\title{
Bericht über die 7. Sitzung des LAGV ALB
}

\author{
(09.-10. Mai 2006 in Bremen)
}

\author{
Dr. A. Hanke
}

SAFGJS, Bahnhofsplatz 29, D-28195 Bremen

- Künftig werden neben dem Stichwortverzeichnis und der Liste der ALB-Beschlüsse auch die genehmigten Protokolle in das FIS-VL eingestellt. Die ALB behält sich vor, die Zugriffsrechte, die zunächst nur auf die ALB-Mitglieder beschränkt bleiben soll, zu erweitern und zu einem späteren Zeitpunkt über eine Aufteilung in einen allgemeinen und einen internen Teil im ALB-Ordner zu entscheiden.

- Unter der Federführung von Niedersachsen wurde ein Eckpunktepapier für einen bundeseinheitlichen Rahmen für die Registrierung von Lebensmittelbetrieben nach der VO (EG) Nr. 852/2004 erarbeitet, das von der ALB zustimmend zur Kenntnis genommen wird.

- Die ALB hat bereits vor längerer Zeit eine Projektgruppe eingerichtet, die sich mit der Auslegung der Amtlichen Berichterstattung nach Art. 14(2) der RL 89/397/EG befasst. Obwohl es eine zum Teil kontroverse Diskussion gab, inwiefern es noch eine Verpflichtung für die EU-Statistik gibt, wird dem Papier große Bedeutung beigemessen, da hiermit die Frage nach der Definition des „Lebensmittelunternehmers“ berührt wird. Das Papier wird noch in einigen wenigen Punkten redaktionell überarbeitet.
In diesem Zusammenhang hat die ALB eine Projektgruppe unter der Leitung von Bayern eingesetzt, die sich mit den Definitionen „Lebensmittelunternehmer“ und „Probe“ beschäftigen wird. Die Notwendigkeit, hier zu einer Klarstellung zu kommen, berührt insbesondere die künftig zu erstellenden Jahresberichte, die zum einen nachvollziehbar und zum anderen länderübergreifend in vergleichbarer Weise erstellt werden sollen.

- Die von der ALB eingesetzten Projektgruppen zu den Konsequenzen der EU-KontrollVO haben zur „Übertragung von Aufgaben“ und zum Thema „Gebühren“ jeweils einen Zwischenbericht vorgelegt. Beide Papiere müssen unter Berücksichtigung der LAGV-Beschlüsse überarbeitet werden.

- Die ALB bittet das BMELV um die Stellungnahme zu den Auslegungen des Lebensmittelbegriffs auch unter Berücksichtigung der Zulassung von Bilanzierten Diäten, die als Infusion verabreicht werden.

- Während der Sitzung konnten noch anteilig Beschlüsse und Arbeitsaufträge der LAGV-Sitzung berücksichtigt werden. Vor dem Hintergrund des Beschlusses der LAGV über die Erstellung der mehrjährigen Kontrollpläne hat die ALB eine Sondersitzung für den 3./4. Juli 2006 eingeplant. 